\title{
SINGULAR HAMILTONIAN SYSTEMS AND SYMPLECTIC CAPACITIES
}

\author{
ALFRED F. KÜNZLE* \\ Max-Planck-Institut für Mathematik \\ Gottfried-Claren-Str. 26, 53225 Bonn, Germany \\ E-mail: kuenzle@mpim-bonn.mpg.de
}

\begin{abstract}
The purpose of this paper is to develop the basics of a theory of Hamiltonian systems with non-differentiable Hamilton functions which have become important in symplectic topology. A characteristic differential inclusion is introduced and its equivalence to Hamiltonian inclusions for certain convex Hamiltonians is established. We give two counterexamples showing that basic properties of smooth systems are violated for non-smooth quasiconvex submersions, e.g. even the energy conservation which nevertheless holds for convex submersions. This also implies that the convexity assumption determines, although not symplectically invariant, a limit case for symplectic geometry. Some applications of this theory are reviewed: symplectic capacities for general convex sets, the symplectic product and a product formula for symplectic capacities.
\end{abstract}

1. Introduction. We consider the linear space $\mathbb{R}^{2 n}$ with the standard closed nondegenerate 2-form $\omega$ as symplectic manifold. One can describe $\omega$ by an almost complex structure $J: T \mathbb{R}^{2 n} \rightarrow T \mathbb{R}^{2 n}, J_{p}^{2}=-i d_{p} \forall p \in \mathbb{R}^{2 n}$, and the standard scalar product. Expressed in coordinate functions to be arranged in analogy to complex ones, $x=\left(x_{1}, \ldots, x_{n}\right)$ with $x_{i}=\left(p_{i}, q_{i}\right), J_{p}$ is given by a constant matrix (denoted again by $J$ )

$$
J=\left(\begin{array}{ccc}
\square & & \\
& \ddots & \\
& & \square
\end{array}\right), \quad \square=\left(\begin{array}{cc}
0 & -1 \\
1 & 0
\end{array}\right) \cong i .
$$

The standard scalar product and the symplectic form are then represented by

$$
x . y=\sum_{i=1}^{2 n} x_{i} y_{i}, \quad \omega(x, y)=J x . y .
$$

1991 Mathematics Subject Classification: Primary 58F05; Secondary 52A20, 58C27, 34A12. Financial support by a fellowship of the Max-Planck-Gesellschaft is gratefully acknowledged.

*On leave at Dépt. de Math., École Polytechnique Fédérale de Lausanne, CH-1015 Lausanne, kuenzle@masg1.epfl.ch

The paper is in final form and no version of it will be published elsewhere. 
A differentiable map is called symplectic if $\varphi^{*} \omega=\omega$, or in the above coordinates if $d \varphi(x)^{T} J d \varphi(x)=J$. We denote the set of symplectic embeddings of open sets in $\mathbb{R}^{2 n}$ into $\mathbb{R}^{2 n}$ by $\mathcal{E}_{\omega}\left(\mathbb{R}^{2 n}\right)$ and the symplectic diffeomorphisms of $\mathbb{R}^{2 n}$ by $\mathcal{D}_{\omega}\left(\mathbb{R}^{2 n}\right)$.

Let $B(r)=B^{2 n}(r)=\left\{x \in \mathbb{R}^{2 n}|| x \mid \leq r\right\}$ be the ball and $Z(r)=B^{2}(r) \times \mathbb{R}^{2 n-2}=$ $\left\{x \in \mathbb{R}^{2 n} \mid q_{1}^{2}+p_{1}^{2} \leq r^{2}\right\}$ be a cylinder with a symplectic base disc.

Now we can state two theorems about symplectic embeddings and diffeomorphisms as motivation for our considerations, quoted in $\mathbb{R}^{2 n}$ only although they hold for general symplectic manifolds.

TheOrem A [G 85]. If the ball B(r) can be symplectically embedded into the cylinder $Z(R)$, then $r \leq R$.

In other words, $B(r)$ cannot be squeezed symplectically into an infinitely long cylinder $Z(R)$ if $r>R$, whereas this can be done for $r \leq R$ by the identity map, expressing a rigidity of symplectic embeddings. Gromov showed that a symplectic invariant defined with $J$-holomorphic curves is the obstruction for such embeddings. It is different from the well known Liouville volume $\operatorname{vol}(D)=\int_{D} \omega^{n}$ which cannot be the obstruction because $\operatorname{vol}(B(r))<\infty$ and $\operatorname{vol}(Z(R))=\infty$, independently of $r$ and $R$.

Such an invariant, nowadays called symplectic capacity, or more precisely symplectic capacity for $\mathcal{F}$ and $\mathcal{D}_{\omega}$ is a map $c$ of a family $\mathcal{F}$ of subsets of $\left(\mathbb{R}^{2 n}, \omega\right)$ to $\mathbb{R}_{+}$satisfying (assuming $D, D^{\prime}, B(r), Z(r) \in \mathcal{F}$ )

(a) $D \subset D^{\prime} \Rightarrow c(D) \leq c\left(D^{\prime}\right)$,

(b) $\varphi \in \mathcal{D}_{\omega} \Rightarrow c(\varphi(D))=c(D)$,

(c) $c(B(r))=\pi r^{2}=c(Z(r))$.

The axioms are designed in the way that the existence of a symplectic capacity implies readily theorem A. The next theorem is another consequence of the existence of a symplectic capacity.

THEOREM B [G85], [E187]. The set of symplectic diffeomorphisms of $\mathbb{R}^{2 n}$ is closed in the set of diffeomorphisms of $\mathbb{R}^{2 n}$ with respect to the $C^{0}$-compact-open topology.

In other words, a topological rigidity is stated. This raised the question whether one can define notions of $C^{0}$-symplectic manifolds and maps as a framework for symplectic topology.

Towards the notion of $C^{0}$-symplectic manifolds, a step has been done in [K90], namely the case of convex sets with non-smooth boundaries, which we report in the present article.

In order to extend the notion of symplectic maps to non-differentiable continuous maps, one can, for a given capacity $c$, consider a subgroup $G_{c}$ of homeomorphisms of $\mathbb{R}^{2 n}$ conserving the capacities of a certain class of subsets of $\mathbb{R}^{2 n}$. Consider for example for $\mathcal{F}$ the set of sub-level sets $S_{q}=\{x \mid q(x)<1\}$ where $q$ varies over the set of all quadratic forms. Let $B$ be the open ball $\left\{\left.x|| x\right|^{2}<1\right\}$, then it is possible to formulate the local symplectic rigidity theorem.

TheOREM C [EH 89]. Let $\varphi_{k}$ in $C^{0}\left(B, \mathbb{R}^{2 n}\right)$ conserve the capacities $c\left(\varphi_{k}\left(S_{q}\right)\right)=c\left(S_{q}\right)$ for all quadratic forms $q$ and converge to $\varphi$ in the sup-norm. Then, for every point $x$ 
where $d \varphi(x)$ exists, $d \varphi(x)$ is either symplectic or antisymplectic:

$$
d \varphi(x)^{T} J d \varphi(x)= \pm J .
$$

Images of smooth hypersurfaces by such maps are non-smooth in general. It is possible to explain, by the theory presented in this article, what Hamiltonian dynamics on such singular hypersurfaces means.

Hamiltonian dynamics is in fact related to symplectic capacities: Ekeland and Hofer showed in their papers [EH89] and [EH90] that both the embedding and topological rigidy can also be understood by means of periodic solutions of Hamiltonian systems instead of $J$-holomorphic curves. We give a very short review of their construction.

For any bounded domain $D \subset \mathbb{R}^{2 n}$ and any function $H$ from the following restricted set

$$
\begin{aligned}
\mathcal{H}(D)=\left\{H \in C^{3}\left(\mathbb{R}^{2 n}, \mathbb{R}_{+}\right) \mid\right. & \exists \text { open } U \supset \bar{D} \text { such that }\left.H\right|_{U}=0, \\
& \left.H(x)=a|x|^{2} \text { for } x \text { large, } a>\pi, a \notin \mathbb{N} \pi\right\},
\end{aligned}
$$

Ekeland and Hofer construct a minimax critical value $c(H)$ of the Hamiltonian action functional $\phi_{H}(x)=\frac{1}{2} \int_{0}^{1} J x(t) \cdot \dot{x}(t) d t-\int_{0}^{1} H(x(t)) d t$ on the space of loops $E:=$ $H^{1 / 2}\left(S^{1}, \mathbb{R}^{2 n}\right)$. This value corresponds to a 1 -periodic solution of the Hamiltonian equation $\dot{x}=J H^{\prime}(x)$ running somewhere in $D^{c}$. One shows the monotonicity $H_{1} \geq H_{2} \Rightarrow$ $c\left(H_{1}\right) \leq c\left(H_{2}\right)$. The real number

$$
c_{E H}(D):=\inf _{H \in \mathcal{H}(D)} c(H)
$$

being independent of $H$, is a good candidate for a symplectic "size" of the set $D$ : In fact $c_{E H}$ is shown to satisfy (a), (b) and (c), the axioms of symplectic capacities.

One would like to be able to define the capacity with the ideal limit Hamiltonian $I_{D}$, the characteristic function of $D$ with value 0 on $D$ and $\infty$ elsewhere, which satisfies $I_{D} \geq H \forall H \in \mathcal{H}_{D}$; then no infimum on Hamiltonian functions had to be taken: a simplification which also needs non-smooth Hamiltonian systems. This application is presented in sections 3.1 and 9 and was one of the basic ideas for [K90].

It is not evident that the Hamiltonian equation should pass to the above infimum. But it does so in the case where the hypersurface $\partial D$ is $C^{1}$ and of restricted contact type, which means that it is regularly submersed in a family of hypersurfaces $\{H(x)=E\}$ generated by a transversal $\omega$-contraction, $L_{X} \omega=\omega$ : The solutions of different levels are equivalent through the contraction, i.e. existence of a solution on a nearby level means existence on $\partial D$.

In this situation (containing the special case of convex hypersurfaces) Ekeland and Hofer get a useful representation result for the capacity: $c_{E H}(D)$ is a multiple of the symplectic action $A(x)=\frac{1}{2} \int \dot{x}(t) . J x(t) d t$ of a $T$-periodic solution $x$ (with unknown $T)$ of $\dot{x}=J H^{\prime}(x)$ on the level $\partial D$, i.e. $c_{E H}(D)=n A(x)$ for some unknown $n \in \mathbb{N}_{+}$, representing the action of an $n$-fold iteration of $x$.

The factor $n$ could not be controlled in [EH 89] and [EH 90], even not in the case of a smooth convex domain $D$ where one conjectured $n=1$. This has been done by Sikorav in August 1990 for the Ekeland-Hofer capacity $c_{E H}$, at least for smooth convex sets. Before and independently, a converse statement has been shown in [K90], namely that the 
least characteristic action $c_{0}$ on convex hypersurfaces is a symplectic capacity for possibly non-smooth convex domains in $\mathbb{R}^{2 n}$. To this aim we studied Hamiltonian type differential inclusions as presented in sections 2 and 3 and used the dual Hamiltonian functional. In another, different approach, Hofer and Zehnder [HZ90] introduced a capacity $c_{E Z}$, whose restriction to smooth convex sets is also equal to $c_{0}$, i.e. $c_{E H}=c_{E Z}=c_{0}$ on smooth convex sets.

A further motivation is the idea that symplectic capacities are possibly calculable by lower and upper sums in analogy to measure theory. This would be an important tool, because capacities are quite difficult to calculate on examples. The idea is to determine the capacity of special cubes, which has been done in [K90], and then give a lower bound for the capacity of a general set $D$ by calculating the capacity of a disjoint union of cubes contained in $D$. But also for this construction, one juxtaposes - most economically - domains with corners and therefore one needs an examination of Hamiltonian dynamics on non-smooth hypersurfaces.

Moreover, one hopes that it would be easier to determine the Hamiltonian dynamics on piecewise linear (PL) hypersurfaces in order to increase the number of known examples.

In fact, the capacity $c_{0}$ can be calculated for symplectic products, see [K90]. To obtain this, we introduced a characterization of closed characteristics on products, which implies a symplectic characterization of polydiscs, see [K95a]. Products having evidently non-smooth boundaries present an other motivation for the present article.

As a summary, we showed in [K90] that a capacity on convex sets can be obtained without an infimum on a set of Hamilton functions and without approximations of $\partial K$ and solutions by a transversal flow. Moreover, no iterated solutions $(n \neq 1)$ interfere. For the monotonicity (b), we used the equivalence of Hamiltonian and characteristic differential inclusion in the convex case (see §3.1) to get an analytical definition by the minimum of the dual Hamiltonian functional, an approach which is simpler than the one in [EH89] but is restricted to convex sets.

In sections 2 to 7 , the main results of this paper are presented. The last three sections consist of a survey of applications to symplectic capacities and symplectic products. More details on these applications are given in [K95] and [K95a].

In [K91], the capacity $c_{0}$ on convex sets is extended to all subsets in $\mathbb{R}^{2 n}$. We got, using [EH90] together with [Si90], or alternatively [EZ90], two distinct symplectic capacities $u$ and $\ell$ which are shown to present an upper and lower bound for all capacities extending the least action on convex sets. For instance the Ekeland-Hofer and Hofer-Zehnder capacities are estimated above and below by $u$ and $\ell$ and can be calculated in certain cases by means of these. The observation that $u$ and $\ell$ are distinct has two interesting consequences: lower and upper sums do not converge to the same value in general, and one conjectures that $c_{E H}$ is different from $c_{H Z}$.

As a simple corollary, two inequalities by Ekeland and Croke-Weinstein respectively are improved.

Another result is for example that all sets $D$ with $B(r) \subset D$ having a periodic orbit (in the general sense of $\S \S 2$ and 8 ) on $\partial D$ with action strictly less than $\pi r^{2}$ cannot be symplectomorphic to a convex set, see [K91]. 
A rule for the capacities of some unions and differences of sets is determined, showing that a capacity does not behave like a measure theory. Moreover isotropic tori and sets with codimension 1 are calculated. Until [K90], no examples other than the ball, the cylinder $Z(r)$ and the ellipsoid which are trivial as they are given by or follow from properties (a), (b) and (c), were known.

2. Characteristic differential inclusions. In order to explain what we mean by Hamiltonian type systems with non-differentiable Hamilton functions, we introduce the characteristic differential inclusion of a Lip-submersed hypersurface $S$. To do this we recall first the differentiable case.

If $H \in C^{1}$ and $S:=\{H(x)=1\}$ is regular, $H^{\prime}(x) \neq 0 \quad \forall x \in S$, one can show that

$$
\dot{x}(t)=J H^{\prime}(x(t)), \quad H(x(0))=1,
$$

has the same trajectories as the so called "characteristic equation" of the hypersurface $S$ with outward normal $n(x), x \in S$ :

$$
\dot{x}(t)=\operatorname{Jn}(x(t)) \quad \text { with } x(t) \in S \forall t .
$$

The equation $(C)$ is characteristic for $S$ in the sense that the set of its solutions (also called "characteristic curves" or "characteristics") is determined by the hypersurface only, more precisely by the restriction of the symplectic structure to $S$, and does not depend on the Hamilton function $H$ generating $S$ as regular hypersurface. Its solutions are parametrizations by arc length of the integral leaves of the field $\operatorname{ker} \omega_{\mid S}$. Two solutions $x_{1}$ and $x_{2}$ of $(H)$ for different Hamilton functions $H_{1}$ and $H_{2}$ are called geometrically equivalent if $\operatorname{Im} x_{1}=\operatorname{Im} x_{2} ;(C)$ together with the choice of an initial point fixes a representative of every equivalence class.

To show the equivalence $(H) \Leftrightarrow(C)$, we just used some basic properties of smooth Hamiltonian systems, which however are violated see $\S \S 5$ to 7 - for non-smooth systems. In spite of these problems, a similar equivalence is proved for convex non-smooth systems in $\S 3.1$.

Our aim is to introduce a natural characteristic equation in the case where $S$ is submersed by a Lipschitzian function.

First, the hypersurface may have edges and corners, where the outward normal is not unique (i.e. a set valued function). Such a generalized normal can be defined if $S$ is given by a Lipschitz function, in particular if $S$ is the boundary of a convex set.

For the last case, the idea is to take, at a given point $x$, all normals of the hyperplanes passing through $x$ whose negative half spaces contain the convex set.

Definition. The (outward) normal cone and the normalized (outward) normal set of a convex set $K$ at a point $x \in \mathbb{R}^{2 n}$ are given by

$$
\begin{aligned}
& N_{K}(x)=\left\{p \in \mathbb{R}^{2 n} \mid p .(x-y) \geq 0 \forall y \in K\right\}, \\
& n_{K}(x)=\left\{p \in N_{K}(x)|| p \mid=1\right\} .
\end{aligned}
$$

It is easy to see that $N_{K}(x)$ is a cone for all $x$, i.e. $p \in N_{K}(x) \Rightarrow \lambda p \in N_{K}(x) \quad \forall \lambda \in$ $\mathbb{R}_{+}$, and that $N_{K}(x)=\{0\}, n_{K}(x)=\emptyset$ for all $x \in \stackrel{\circ}{K}$. Furthermore, for a smooth point $x$ of $\partial K, N_{K}(x)=\mathbb{R}_{+} n, n_{K}(x)=\{n\}$, where $n$ is the usual normalized outward normal 
vector at $x$. One sees readily that $N_{K}$ is a set valued vector field in $K$ with support on $\partial K$, thus suitable for our aim to construct a system which is characteristic for the hypersurface $\partial K$.

Secondly, it is clear that for instance curves passing through a corner of $\partial K$ cannot be differentiable. The idea is to ask that $\gamma$ should be differentiable only almost everywhere. One would therefore like to study

$$
\text { (i) } \dot{\gamma}(t) \in J n_{K}(\gamma(t)) \text { a.e., }
$$

where a solution $\gamma$ is asked to be parametrized by arc length and Lipschitzian with constant 1: $\left|\gamma(t)-\gamma\left(t^{\prime}\right)\right| \leq \int_{t^{\prime}}^{t}|\dot{\gamma}(t)| d t=\left|t-t^{\prime}\right|$; Lipschitzian functions are almost everywhere differentiable (by Rademacher's theorem), so that the system is consistent. Here it is already plausible that the non-uniqueness problem is present, because $n_{K}(x)$ is set valued and also $J n_{K}(x)$ may intersect the generalized tangent space of $\partial K$ in a (non-trivial) family of directions.

But is not clear yet whether a submersed family of convex hypersurfaces would define a field with energy conserving solutions. The answer is given in section 5: It is negative.

Therefore, a natural idea to generalize $(C)$ is to consider $(i)$ together with the additional constraint $(i i)$ which is automatically satisfied in the smooth case, see $\S 3$. It is called characteristic differential inclusion for $\partial K$ :

(i) $\dot{\gamma}(t) \in J n_{K}(\gamma(t))$ a.e.,

(ii) $\gamma(t) \in \partial K \quad \forall t \in\left[0, T_{\gamma}\right]$.

3. Hamiltonian inclusions. Some new facts on convex Hamiltonian systems are established in this section.

Definition. The subdifferential of a locally Lipschitz function $H$ is defined by

$$
\partial H(x)=\left\{p \in \mathbb{R}^{2 n} \mid \forall v \in \mathbb{R}^{2 n} p . v \leq D_{+} H(x)(v):=\lim _{h \rightarrow 0^{+}} \frac{H(x+h v)-H(x)}{h}\right\} .
$$

Its elements are called subgradients. $x$ is called a critical point of $H$ if $0 \in \partial H(x)$.

For convex functions, $\partial H(x)$ is non-empty and bounded for all $x \in \mathbb{R}^{2 n}$ (see [A84]). At a point where $H$ is differentiable, $\partial H(x)$ reduces to $\left\{H^{\prime}(x)\right\}: p . v \leq H^{\prime}(x) . v \forall v \in \mathbb{R}^{2 n} \Rightarrow$ $p=H^{\prime}(x)$.

The subdifferential is thus a generalization of the gradient in the same way as the normal cone generalizes the outward normal. In fact, we will show now that, for some convex "gauge functions" $H$ of a bounded convex domain $K, \partial H(x)$ and $n_{K}(x)$ differ only by the length of their elements.

3.1. Equivalence. For convex sets $K$ containing 0 in their interior we now choose $H(x)=\left(j_{K}(x)\right)^{\alpha}$ as Hamilton function, where $j_{K}(x)=\inf \left\{\lambda \mid \frac{x}{\lambda} \in K\right\}$ is the so called gauge function of $K$, and for $\alpha$ we assume $\alpha \geq 1$. $H$ is convex, finite and $\alpha$-homogeneous. Moreover $\stackrel{\circ}{K}=\{x \mid H(x)<1\}$.

With this choice of $H$ the following fixed energy problem is equivalent to $(C I)$ :

$$
\left\{\begin{array}{l}
-J \dot{x}(t) \in \partial H(x(t)) \quad \text { a.e. } \\
H(x(t))=1 \quad \forall t \in[0, T]
\end{array}\right.
$$


where $x(t)$ is assumed Lipschitz.

TheOrem 1 (Equivalence of $(C I)$ and $(H I)$ ). For $H$ as above:

(a) $n \in N_{K}(x) \Leftrightarrow \frac{\alpha}{n \cdot x} n \in \partial H(x)$ for $x \in \partial K$,

(b) $(C I)$ and $(H I)$ have the same solutions up to monotone absolutely continuous reparametrizations.

Proof. (a) Observe beforehand that there is a small ball $B_{\varepsilon}(0) \subset K$, therefore there is $r>0$ such that $r x \in K \quad \forall x \in \partial K$, and conclude $n . x \geq n . r n>0$ for all non-zero $n \in N_{K}(x)$ and for all $x \in \partial K$. Division by $n . x$ is then well-defined; in the case $n=0$, the quotient $\frac{n . y}{n \cdot x}$ is defined to be 0 by continuity.

As a first step, we prove the equivalence (for $x \in \partial K$ ):

$$
n \in N_{K}(x) \Leftrightarrow j_{K}(y) \geq \frac{n \cdot y}{n \cdot x} \forall y \in \mathbb{R}^{2 n},
$$

namely

$$
\begin{aligned}
n \in N_{K}(x) & \Leftrightarrow n \cdot(x-y) \geq 0 \forall y \in K \\
& \Rightarrow n \cdot(x-y) \geq 0 \forall y \in \partial K \\
& \Leftrightarrow n \cdot\left(x-\frac{y}{j_{K}(y)}\right) \geq 0 \forall y \in \mathbb{R}^{2 n} \\
& \Leftrightarrow j_{K}(y) \geq \frac{n \cdot y}{n \cdot x} \forall y \in \mathbb{R}^{2 n} .
\end{aligned}
$$

Conversely, using $1 \geq j_{K}(y) \forall y \in K$, we get

$$
\begin{aligned}
j_{K}(y) \geq \frac{n \cdot y}{n \cdot x} \forall y \in \mathbb{R}^{2 n} & \Rightarrow 1 \geq \frac{n \cdot y}{n \cdot x} \forall y \in K \\
& \Leftrightarrow n \in N_{K}(x) .
\end{aligned}
$$

Therefore the above implications are equivalences.

As a second step, we apply the equivalence (true because $H$ is convex)

$$
p . x-H(x)=\min _{y \in \mathbb{R}^{2 n}}(p . y-H(y)) \Leftrightarrow p \in \partial H(x)
$$

(Legendre duality) for the choice $p=\frac{1}{n \cdot x} n$ with given $n \in N_{K}(x)$ :

$$
\begin{aligned}
n \in N_{K}(x) & \Leftrightarrow 0 \geq \frac{n \cdot y}{n \cdot x}-j_{K}(y) \forall y \in \mathbb{R}^{2 n} \\
& \Leftrightarrow \frac{1}{n \cdot x} n . x-H(x)=0 \geq \frac{1}{n \cdot x} n . y-j_{K}(y) \forall y \in \mathbb{R}^{2 n} \\
& \Leftrightarrow \frac{1}{n \cdot x} n \in \partial j_{K}(x) .
\end{aligned}
$$

Finally, the right derivative of $H(x)=\left(j_{K}(x)\right)^{\alpha}$ for $\alpha \geq 1$ is given by

$$
D_{+} H(x)(v)=\alpha\left(j_{K}(x)\right)^{\alpha-1} D_{+} j_{K}(x)(v) \quad \forall v \in \mathbb{R}^{2 n},
$$

its subdifferential is therefore $\partial H(x)=\alpha \partial j_{K}(x)$, from where we conclude

$$
n \in N_{K}(x) \Longleftrightarrow \frac{\alpha}{n . x} n \in \partial H(x) .
$$


It is remarkable that $N_{K}(x)$ is a cone whose rays correspond to exactly one element of $\partial H(x)$, namely $p=\alpha \frac{n}{n \cdot x}$. By normalization we get

$$
n \in n_{K}(x) \Leftrightarrow \frac{\alpha}{n \cdot x} n \in \partial H(x) \text { and }|n|=1 .
$$

(b) Problems $(H I)$ and $(C I)$ are restricted to the same energy hypersurface $\partial K$, so that (a) applies:

$$
\begin{aligned}
& -J \dot{x}(t) \in \partial H(x(t)) \Rightarrow \frac{-J \dot{x}(t)}{|-J \dot{x}(t)|} \in n_{K}(x(t)), \\
& -J \dot{\gamma}(s) \in n_{K}(\gamma(s)) \Rightarrow \frac{\alpha}{-J \dot{\gamma}(s) \cdot \gamma(s)} \cdot(-J \dot{\gamma}(s)) \in \partial H(\gamma(s)),
\end{aligned}
$$

where vanishing numerators are excluded. The reparametrizations are thus given by the monotone functions $\tau$ and $\tau^{-1}$ :

$$
\begin{array}{rlrl}
\tau(t) & =\int_{0}^{t} \frac{\alpha}{-J \dot{\gamma}(s) \cdot \gamma(s)} d s, & x(t):=\gamma(\tau(t)), \\
\tau^{-1}(s)=\int_{0}^{s} \frac{1}{|\dot{x}(t)|} d t, & \gamma(s):=x\left(\tau^{-1}(s)\right) .
\end{array}
$$

If we fix the domain of $\tau$ to be $\left[0, T_{\gamma}\right]$, then the minimality of the period $T_{x}$ follows from the minimality of $T_{\gamma}$, and conversely. It is obvious that $\tau$ and $\tau^{-1}$ are absolutely continuous.

Remark. Observe that also the characteristic function of a set

$$
I_{K}(x)= \begin{cases}0 & \text { if } x \in K \\ \infty & \text { otherwise }\end{cases}
$$

and the normal cone are related: $\partial I_{K}(x)=N_{K}(x)$. This is (morally) used for symplectic capacities as explained in $\S 9$.

3.2. Energy conservation. After we showed that there exist non-differentiable quasiconvex Hamilton functions having solutions which do not conserve the energy (cf. section 5), I. Ekeland asked R. T. Rockafellar whether this would happen with convex functions also. The answer was the following theorem, the proof of which is given and reformulated for convenience. An earlier proof can be found in [Cl80], as pointed out by F. Clarke.

THEOREM 2 [R89]. Let $H$ be a finite convex function from $\mathbb{R}^{2 n}$ to $\mathbb{R}$ and $x \in$ $W^{1,1}\left([0, T], \mathbb{R}^{2 n}\right)$ a solution of

$\left(H_{0}\right) \quad \dot{x}(t) \in J \partial H(x(t)) \quad$ a.e. in $[0, T]$.

Then $H(x(t))=H(x(0)) \forall t \in[0, T]$.

Proof. A solution $x$ is Lipschitzian with a Lipschitz constant bounded by $\max \{|p| \mid$ $p \in \partial H(x(t)), t \in[0, T]\}<\infty$ and $H$ is locally Lipschitzian by [A84, p. 19-21]. Therefore $\theta(t)=H(x(t))$ is locally Lipschitzian and thus almost everywhere differentiable (Rademacher). With this regularity, one can establish $\dot{\theta}(t)=0$ almost everywhere; this is done in an analogous way as in the $C^{1}$-case: Let us denote by $\mathcal{T}$ the set of full measure 
where $\theta(t)$ and $x(t)$ are differentiable and $\dot{x}(t) \in J \partial H(x(t))$. For all $t \in \mathcal{T}$, the right and left derivatives coincide, in particular for $v=\dot{x}(t)$ :

$$
\dot{\theta}(t)=D_{+} H(x(t))(\dot{x}(t))=-D_{+} H(x(t))(-\dot{x}(t)) .
$$

Because of $-J \dot{x}(t) \in \partial H(x(t))$, we get

$$
-J \dot{x}(t) \cdot v \leq D_{+} H(x(t))(\dot{x}(t))
$$

for both $v= \pm \dot{x}(t)$. The antisymmetry of $J$ yields

$$
\begin{aligned}
& 0=-J \dot{x}(t) \cdot \dot{x}(t) \leq D_{+} H(x(t))(\dot{x}(t)), \\
& 0=-J \dot{x}(t) \cdot \dot{x}(t) \geq D_{+} H(x(t))(\dot{x}(t)) .
\end{aligned}
$$

Therefore $D_{+} H(x(t))(\dot{x}(t))=0=\dot{\theta}(t) \forall t \in \mathcal{T}$ and $\theta(t)=H(x(t))=$ const.

4. Quasiconvex submersions. Now consider a family of sets $K(s) \subset \mathbb{R}^{N}, s \in \mathbb{R}$, continuous with respect to the Hausdorff metric on sets.

Definition. A family $K(s)$ is called regular if $\partial K(s) \cap \partial K\left(s^{\prime}\right)=\emptyset, \forall s \neq s^{\prime}$.

This generalizes the notion of a $C^{1}$-submersed family of hypersurfaces to the nondifferentiable case. In fact, it implies that $\partial K(s)$ can be given as levels of a continuous function $f: \bigcup_{s} K(s) \rightarrow \mathbb{R}$ defined by

$$
f(x)=s \Leftrightarrow x \in K(s) .
$$

If $f$ is $C^{1}$, then $f^{\prime}(x) \neq 0 \quad \forall x \in K(s)$, which is the classical definition of the regularity of the hypersurface $K(s)$.

Now the relation between $f$ and $K(s)$ is examined if $K(s)$ consists of convex sets.

Definition. A function $f$ and the family $\{x \mid f(x)=s\}_{s \in \mathbb{R}}$ are called quasiconvex if the sublevels $\{x \mid f(x) \leq s\}$ are convex for all $s \in \operatorname{Im} f$. The family $K(s)$ or $\partial K(s)$ is called convexifiable in the interval $I$ if there is a convex function $H: \mathbb{R}^{N} \rightarrow \mathbb{R}$ and a reparametrization $h: I \rightarrow \mathbb{R}$ such that

$$
\partial K(s)=\left\{x=\mathbb{R}^{N} \mid H(x)=h(s)\right\} \quad \forall s \in I .
$$

It is easy to give quasiconvex families which are convexifiable, e.g. $f(r)=\sqrt{r}$ or $f(r)=$ $\sqrt{\sup _{i}\left|x_{i}\right|}$. In section 5 , we present a quasiconvex example which is not convexifiable, together with a proof of this property using the energy conservation of Hamiltonian inclusions as established in theorem 2.

5. A quasiconvex example (CE1). Let $c(s)=\left(\begin{array}{c}-\sin s \\ -1+\cos s\end{array}\right)$ be the parametrization by arc length of the unit circle with center $\left(\begin{array}{c}0 \\ -1\end{array}\right)$ and $n_{s}:=\left(\begin{array}{c}-\sin s \\ +\cos s\end{array}\right)$ the outward unit normal. Then consider the family

$$
K(s):=\left\{x \in \mathbb{R}^{2}|| x|\leq| c(s) \mid \text { and } n_{s} . x \leq 0\right\}, \quad s \in[0, \pi],
$$

of convex sets given by intersection of halfplanes and discs of radii $|c(s)|=\sin \frac{s}{2}$. 


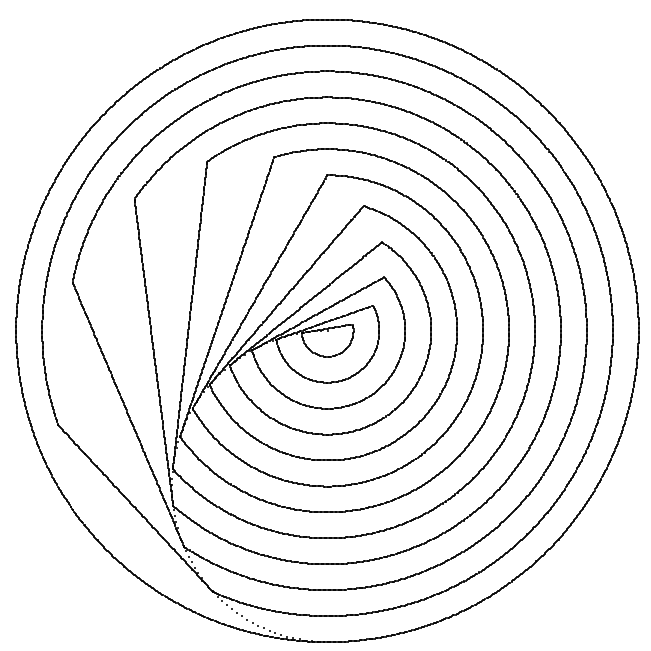

Fig. 1. A quasiconvex family in $\mathbb{R}^{2}$ violating energy conservation (CE1)

It is easy to show that the distance between the boundaries is positive:

$$
\operatorname{dist}\left(\partial K\left(s^{\prime}\right), \partial K(s)\right)=2\left(\sin \frac{s^{\prime}-s}{2}\right)^{2}>0 \quad \forall s, s^{\prime} \in[0, \pi], s^{\prime}>s,
$$

which means that $\partial K(s)$ are mutually disjoint and $K(s)$ defines a regular family of convex sets: For all $x \in B(2)=K(\pi)$ there is a unique value $s=: f(x)$ such that $x \in \partial K(s)$. One gets the representation of $K(s)$ by

$$
\{f(x) \leq s\}=K(s)
$$

$f$ is a quasiconvex function which is at least of class $C^{0}$ (by the continuity of $n_{s}$ and $|c(s)|$ in $s$ ) but not $C^{1}$ because there are two curves of corners, the first one being $c(s)$.

The characteristic differential inclusion adapted to the family $K(s)$ is

$$
\dot{\gamma}(t) \in J n_{K(E(t))}(\gamma(t)) \quad \text { a.e., }
$$

which has to be solved for $(E, \gamma) \in C^{0}([0, T],[0, \pi]) \times \operatorname{Lip}([0, T], B(2))$ including the a priori unknown energy function.

We examine two special solutions:

(i) The parametrization by arc length $\gamma_{s}$ of $\partial K(s)$, for $s$ fixed, defines a solution $\left(\gamma_{s}, s\right)$ with constant energy $E(t)=s$. The inclusion (1) is in fact solved for all but two exceptional points; one observes moreover that the right and left derivatives satisfy it even everywhere.

(ii) The corner curve $c(s)$ is another solution for $t \in] 0, \pi]$. It satisfies (1) at every time $t$ although it consists of corners only:

$$
\left.\left.\frac{d}{d t} c(t)=\left(\begin{array}{l}
-\cos t \\
-\sin t
\end{array}\right)=J n_{t} \in J n_{K(E(t))}(c(t)) \quad \forall t \in\right] 0, \pi\right]
$$

with $E(t)=t$. But the solution $c$ does not conserve the "energy"! 
As a consequence of (i) and (ii), one concludes that the initial value problem (IVP) at $x_{0}=c(s)$ possesses at least two solutions, $\gamma_{s}\left(t+s_{0}\right)$ and $c(t+s)$. In fact, there are infinitely many geometrically different solutions $(\gamma, E)$ for

$$
\dot{\gamma}(t) \in J n_{K(E(t))}(\gamma(t)), \quad \gamma(0)=x_{0},
$$

for every initial point $x_{0} \in \mathcal{A}:=\operatorname{int}(B(2) \backslash\{0\})$.

Moreover, the boundary value problem for $x_{0}, x_{1} \in \mathcal{A}$

$$
\dot{\gamma}(t) \in J n_{K(E(t))}(\gamma(t)), \quad \gamma(0)=x_{0}, \quad \gamma(T)=x_{1},
$$

has infinitely many solutions $(\gamma, E, T)$ if and only if $f\left(x_{0}\right) \leq f\left(x_{1}\right)$.

One finds that the function $E(t)$ is increasing for any solution $(\gamma, E)$. This is due to the special property of our example that $J n \in J n_{K}$ points to the exterior of $K$. Consequently, every solution for the boundary values $x_{0}, x_{1}$ on the same level $\partial K(s)$ conserves the energy $f: f\left(x_{0}\right)=f\left(x_{1}\right) \Leftrightarrow E(t)=$ const $=f\left(x_{0}\right)$. Whence all periodic solutions of (1) conserve the energy in this special example.

Now we apply the theorem of Rockafellar: If there were a convex function $k$ parametrizing $K(s)$, then all solutions of (HI) would conserve the energy and (HI) would be equivalent to (1) by theorem 1 , which is a contradiction. This implies that CE1 is not convexifiable in $[0, \pi]$. We remark that this result is even more general than what can be obtained by classical methods of convex analysis, see the proof of Crouzeix [C89] as reported in [K90] that CE1 is not convexifiable in $\left[0, \frac{\pi}{2}\right]$.

The study of singular Hamiltonian systems therefore provides a new tool to decide whether a given family of convex sets in even dimensions is convexifiable or not.

6. A convex example (CE2). So far we have constructed a quasiconvex system whose solutions of IVP and BVP may not be unique for certain points and at the same time do not always conserve the energy; now we show that even in convex systems, where the energy is always conserved, the IVP and BVP may have an infinity of solutions.

Proposition 1. All isotropic edges of dimension $\geq 2$ of the standard cube in $\mathbb{R}^{2 n}$ have infinitely many solutions of the IVP at all its interior points. The BVP of a pair of points has infinitely many solutions if the second point lies in a characteristic cone of attainable points.

Pr o of. We study the fixed energy initial value problem

$$
\begin{aligned}
& \dot{\gamma}(t) \in J n_{K(s)}(\gamma(t)) \quad \text { a.e., } \\
& \gamma(t) \in \partial K(s), \quad s \text { fixed, } \\
& \gamma(0)=x_{0} .
\end{aligned}
$$

It is enough to study a 4-dimensional cube, which can be considered as a (restricted symplectic) edge of a higher dimensional one.

Let $K(s)=\left\{\left(p_{1}, q_{1}, p_{2}, q_{2}\right) \in \mathbb{R}^{4} \mid p_{i}, q_{i} \in[0, s], s \in \mathbb{R}_{+}\right\}$be the cubes with side lengths $s$. Its boundary $\partial K(s)$ is composed of edges (strata) of dimension 0 (corners), 1 , 2 and 3 (faces). At an interior point of such an edge, the set of normalized normals is 3 , 2, 1 and 0-dimensional respectively. 


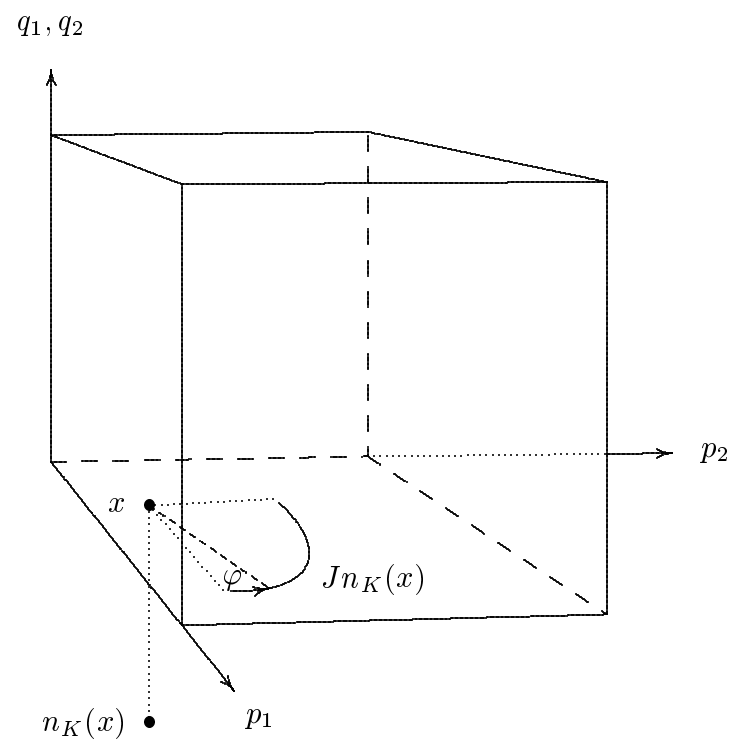

Fig. 2. Non-uniqueness in isotropic edges (CE2)

To give a counterexample against the uniqueness on any energy level, consider for example the 2-dimensional isotropic (Lagrangian) edge

$$
A^{2}=\left\{\left(p_{1}, 0, p_{2}, 0\right) \mid p_{i} \in[0,2]\right\}
$$

of $K:=K(2)$, see fig. 2. The set of normalized normals for an interior point $x \in \operatorname{int} A^{2}$ is

$$
n_{K}(x)=\{(0,-\cos \varphi, 0,-\sin \varphi) \mid \varphi \in[0, \pi / 2]\} .
$$

After application of $J$ we get

$$
J n_{K}(x)=\{(\cos \varphi, 0, \sin \varphi, 0) \mid \varphi \in[0, \pi / 2]\} .
$$

The set of solutions with initial point $x$ is parametrized by an infinite dimensional function space $\left\{\phi \in C^{0}\left([0, T],\left[0, \frac{\pi}{2}\right]\right)|| \dot{\phi}(t) \mid=1 \forall t\right\}$ :

$$
\gamma_{\phi}(t):=x+(\cos \phi(t), 0, \sin \phi(t), 0),
$$

and the boundary value problem for two points $x, y \in A^{2}$ possesses still infinitely many solutions of this form if $y$ lies in the "attainable cone" of points $y$ satisfying $x-y \in$ $J n_{K}(x)=J n_{K}(y)$.

We remark that $K$ is a symplectic product of two-dimensional squares and moreover a (non-smooth) completely integrable system; a similar behaviour is observed in general symplectic products (see [K94c]) even if the factors have smooth boundary.

COROllary 1. CE2 does not define a flow. Nevertheless, if at every point $x \in A^{2}$ a preferred direction $\in J N_{K}(x)$ is assigned depending smoothly on $x$, we get a flow in $A^{2}$ which preserves the symplectic form. One observes therefore a non-uniqueness of the Hamiltonian flow. 
Proof. This is immediate, because every vector field defines a flow, and the deformation of curves along isotropic edges preserves the symplectic area although the Riemannian area is not conserved.

COROLlary 2 (Regularity). The solutions of $(C I)$ and (HI) may not be differentiable from the right everywhere, whereas a convex function defining $S$ is: The regularity of the solutions is strictly smaller than that of the defining function.

Proof. A curve with $\gamma(0)=x$ consisting of a sequence of connected vertical and horizontal arcs accumulating at $x$ describes a solution of the form $\gamma_{\phi}$ which is not differentiable from the left nor from the right, as the limit

$$
\dot{\gamma}^{+}(t):=\lim _{h \rightarrow 0^{+}} \frac{\gamma(t+h)-\gamma(t)}{h}
$$

does not exist at $t=0$ because it would be composed of two vectors, a horizontal and a vertical one. The maximal regularity one can hope for is strictly less than right differentiability, whereas convex functions are right differentiable.

7. Conclusions. We showed that solutions of $C^{0}$-systems in general

(1) don't conserve the energy (CE1);

(2) don't have unique solutions of the initial value problem $(\mathrm{CE} 1,2)$;

(3) don't have the same regularity as $H(\mathrm{CE} 1,2)$;

(4) depend on the Hamilton function representing the hypersurface as energy level $(\mathrm{CE} 1)$

These are four violations of very basic properties of $C^{1+\varepsilon}$-systems, $\varepsilon>0$, namely of properties which were used to show the equivalence of $(H)$ and $(C)$. The example for (1) could be made quasiconvex but not convexifiable, because of theorem 2 . This reveals that the convex Hamiltonians form a distinguished limit case in symplectic topology, at least in $C^{0}$-category, although convexity is not symplectically invariant. This also means that $(C)$ and $(H)$ don't have equivalent generalizations to the non-smooth case, unless further assumptions on $H$ are considered, namely $H$ e.g. of the form $\left(j_{K}\right)^{\alpha}$ and the explicit additional energy constraint, see $\S 3.1$. We therefore adopt the point of view that the Hamiltonian description is secondary and that one should consider the characteristic equation or inclusion as primary and more geometric.

8. Closed characteristics. To give a geometric definition of a symplectic capacity, we are interested in periodic solutions of the characteristic differential inclusion $(C I)$, called closed characteristics. More precisely, we consider absolutely continuous prime periodic solutions of

$$
\left\{\begin{aligned}
\text { (i) } & \dot{\gamma}(t) \in J n_{K}(\gamma(t)) \quad \text { a.e. } \\
\text { (ii) } & \gamma(t) \in \partial K \quad \forall t \in\left[0, T_{\gamma}\right] \\
\text { (iii) } & \gamma\left(t+T_{\gamma}\right)=\gamma(t) \quad \forall t \in\left[0, T_{\gamma}\right] \\
& \text { and } T_{\gamma}>0 \text { is the minimal period of } \gamma
\end{aligned}\right.
$$


namely the set

$$
\Gamma(K):=\left\{\gamma \in \operatorname{Lip}\left(\left[0, T_{\gamma}\right], \mathbb{R}^{2 n}\right) \mid(*)\right\}
$$

Solutions of $(*)$ have the ambiguity of the choice of an initial value, so for every solution we get an $S^{1}$-orbit in $\Gamma(K)$ with respect to the natural $S^{1}$-action which leaves $(*)$ invariant. We pass to the quotient

$$
\Gamma^{*}(K):=\Gamma(K) / S^{1}
$$

to get the set of geometrically different solutions of $(*) . \Gamma^{*}(K)$ can be regarded as the set of representatives of the moduli space of prime closed characteristic curves.

A symplectic size of loops is given by their symplectic action

$$
A(\gamma)=\frac{1}{2} \int_{0}^{T_{\gamma}} \dot{\gamma}(t) . J \gamma(t) d t .
$$

We will call loops small or big according to the absolute value of $A(\gamma)$, and symplectic actions of elements of $\Gamma(K)$ (prime closed characteristics) are called characteristic actions of $\partial K$. Observe that the parameter transformations preserving orientation and mapping degree conserve the value of $A(\gamma)$, therefore $A(\gamma)$ passes to the quotient by such reparametrizations.

$A$ is not invariant with respect to the action of $\mathbb{N}$ given by the iteration $\gamma \mapsto \gamma^{(k)}$, as $A\left(\gamma^{(k)}\right)=k A(\gamma)$. For convenience we define

$$
\Gamma^{\mathbb{N}_{+}}(K)=\left\{\gamma^{(k)} \mid \gamma \in \Gamma(K), k \in \mathbb{N}_{+}\right\}
$$

as the set of iterated characteristic loops, which by definition don't solve $(*)$. Their minimal period is $\ell\left(\gamma^{(k)}\right) / k$ instead of $\ell\left(\gamma^{(k)}\right)$.

\section{Symplectic capacities}

Definition. Let $\mathcal{K}=\left\{K \subset \mathbb{R}^{2 n} \mid 0 \in \stackrel{\circ}{K}\right.$, convex $\}$ be the set of convex bodies, where $\stackrel{\circ}{K}$ denotes the interior of $K$. Define $c$ to be the map

$$
c: \mathcal{K} \rightarrow \mathbb{R}_{+}, \quad K \mapsto c(K)=\inf \{A(\gamma) \mid \gamma \in \Gamma(K)\},
$$

assigning to $K$ the minimal characteristic action of $\partial K$. We will call $c$ symplectic capacity ${ }^{*}$ as soon as $c(K)$ is shown to be well defined, attained by a $\gamma^{*} \in \Gamma(K)$ for bounded $K$, positive and satisfying the defining properties (a), (b) and (c). By convention, $c(K)=\infty$ if $\Gamma(K)=\emptyset$, which does not occur if $K$ is bounded.

To show that $c(K)$ is positive, we used the gauge function $j_{K}(x)(\S 3)$ and the well known dual Hamiltonian action functional of $H=\left(j_{K}\right)^{\alpha}$, with $\alpha>0$, see [CE80] and [E90]. We proved that $c(K)$ is a monotone function of the minimum of this functional [K90]. The well known fact that the dual functional is bounded from below as opposed to the direct one, is a remarkable advantage of the dual formulation: a minimum can be calculated by a computer whereas saddle points can't in general.

We call this the geometric definition as it defines $c$ independently of Hamiltonian functions by a purely geometric term, the symplectic area of a surface $\Sigma \subset K$ contoured

*It is the one we denoted in the introduction by $c_{0}$. 
by the characteristic loop $\gamma^{*}$ :

$$
A\left(\gamma^{*}\right)=\frac{1}{2} \int_{\operatorname{Im} \gamma^{*}} \gamma^{*} \cdot(-J) d \gamma^{*}=\int_{\Sigma} \omega .
$$

It is independent of the choice of $\Sigma$ with $\partial \Sigma=\operatorname{Im} \gamma^{*}$, because $K$ is contractible. For arbitrary closed curves $\gamma, A(\gamma)$ is a relative integral invariant in terms of Poincaré [P99, p. 5]. Moreover, for closed characteristics $\gamma \in \Gamma^{*}(K), A(\gamma)$ is a fundamental integral for both canonical and geometric quantization and relates classical to quantum mechanics.

Now we collect some properties of $c$.

THEOREM 3 [K90]. The least characteristic action $c$ is a symplectic capacity for convex sets $\mathcal{K}$ and embeddings $\mathcal{E}_{\omega}$ :

(a) $K \subset K^{\prime} \Rightarrow c(K) \leq c\left(K^{\prime}\right)$,

(b) $\phi \in \mathcal{E}_{\omega} \Rightarrow c(\phi(K))=c(K)$,

(c) $c(B(r))=\pi r^{2}=(Z(r))$,

for all $K, K^{\prime} \in \mathcal{K}$. Moreover, the following properties are satisfied:

(d) $c$ is Hausdorff continuous on $\mathcal{K}$,

(e) $c\left(K \times K^{\prime}\right)=\min \left\{c(K), c\left(K^{\prime}\right)\right\}$ for symplectic products $K \times K^{\prime}$,

(f) After continuous extension, $c(K)=0$ for bounded convex sets $K \subset \mathbb{R}^{2 n-1} \subset \mathbb{R}^{2 n}$.

As mentioned in the introduction, Sikorav [Si90] proved that the capacity by Ekeland and Hofer, which was defined to be the infimum of a certain critical value over a set of Hamiltonian functions, equals $c$ on smooth convex bodies and is acheived by a noniterated solution. The characterization by a closed characteristic on non-smooth convex sets was of course not possible there, because the notion of a characteristic system as developed in the present article was not made precise yet.

10. The symplectic product. In [K90], we gave a representation of all characteristic loops on $\partial\left(K_{1} \times K_{2}\right)$ by characteristic loops on the factors $\partial K_{i}$ and conversely. This suggests a product formula for all capacities which are given by actions of characteristic loops. Here we will only indicate what is needed to show formula (e) for the least characteristic action $c$.

Consider two linear symplectic spaces $\left(\mathbb{R}^{2 n_{i}}, \omega_{i}, g_{i}\right)$ with $\omega_{i}$ and $g_{i}$ as in the introduction. In the canonical metric $g=g_{1} \oplus g_{2}$, denoted by $x . y$ again, the splitting of $E=\mathbb{R}^{2 n}=\mathbb{R}^{2 n_{1}} \oplus \mathbb{R}^{2 n_{2}}=E_{1} \oplus E_{2}$ is orthogonal. Consider the orthogonal projections on these factors, expressed in coordinates by $P_{1} x=\left(\begin{array}{c}x_{1} \\ 0\end{array}\right)$ which we identify with $P_{1} x=x_{1}$ for $x=\left(\begin{array}{l}x_{1} \\ x_{2}\end{array}\right)$.

The natural symplectic form is $\omega=\omega_{1} \oplus \omega_{2}$, given by $J=J_{1} \oplus J_{2}$.

Now look at the symplectic product $K:=K_{1} \times K_{2} \subset \mathbb{R}^{2 n_{1}} \times \mathbb{R}^{2 n_{2}}$. We suppose $K_{i} \in$ $\mathcal{K}\left(\mathbb{R}^{2 n_{i}}\right)$ to simplify the proof and to fit the product formula in our convex framework:

$$
K \in \mathcal{K}\left(\mathbb{R}^{2 n}\right) \Leftrightarrow K_{i} \in \mathcal{K}\left(\mathbb{R}^{2 n_{i}}\right) \text { for } i=1 \text { and } 2 .
$$

It is easy to show that $N_{K}\left(x_{1}, x_{2}\right)=N_{K_{1}}\left(x_{1}\right) \oplus N_{K_{2}}\left(x_{2}\right) \forall x=\left(x_{1}, x_{2}\right) \in K_{1} \times K_{2}$ and that the operators $J, P_{i}$ and $d / d t$ commute. 
TheOREM 4. For all $\gamma \in \Gamma\left(K_{1} \times K_{2}\right), P_{i} \gamma$ belongs to $\Gamma^{\mathbb{N}_{+}}\left(K_{i}\right) \cup K_{i}$ up to reparametrization of degree 1 , where $K_{i}$ denotes the set of constant functions with values in $K_{i}$.

R e m a r k. The lack of $C^{1}$-regularity of the solutions of $(*)$ was handled by a careful control of the differential inclusion almost everywhere, more precisely, we considered a representative of the Lebesgue class of the derivative and controlled it pointwise on a set of full measure.

As an alternative approach, we smoothened $K_{1} \times K_{2}$ by Yoshida's approximation and followed the perturbed $C^{1}$-solutions as the perturbation parameter changes. Just as in the situation of Viterbo's proof for contact manifolds [V87], these perturbed solutions approach the hypersurface from the outside. But the notion of the characteristic differential inclusion still has to be made precise and examined for the limit, i.e. with the smoothening approach one does not gain anything.

Remark. A converse statement to theorem 4 can also be proved (see [K90]): Given $\Gamma\left(K_{1}\right)$ and $\Gamma\left(K_{2}\right)$, one recovers $\Gamma\left(K_{1} \times K_{2}\right)$ by an adapted family of parameter transformations. Again, the interesting part is $\partial K_{1} \times \partial K_{2}$, where a non-resonance condition has to be checked. This condition is similar to the usual non-resonance condition on Lagrangian tori; just remark that in fact $\partial K_{1} \times \ldots \times \partial K_{n}$ is a Lagrangian torus in $\mathbb{R}^{2 n}$ if $K_{i}$ are 2-dimensional simply connected subsets of $\mathbb{R}^{2}$.

Remark. The symplectic product is physically non-trivial as it represents a composed system with non-zero interaction. In terms of gauge functions, consider

Use

$$
\begin{aligned}
h_{i}\left(x_{i}\right) & =\left(j_{K_{i}}\left(x_{i}\right)\right)^{2}, \\
H\left(x_{1}, x_{2}\right) & =\left(j_{K_{1} \times K_{2}}\left(x_{1}, x_{2}\right)\right)^{2}=\max \left\{h_{i}\left(x_{i}\right) \mid i=1,2\right\} \\
& =h_{1}\left(x_{1}\right)+h_{2}\left(x_{2}\right)+V_{1,2}\left(x_{1}, x_{2}\right) .
\end{aligned}
$$

$$
\begin{aligned}
j_{K_{1} \times K_{2}}\left(x_{1}, x_{2}\right) & =\inf \left\{\lambda \in \mathbb{R}_{+} \mid x / \lambda \in K\right\}=\sup \left\{\lambda \mid \lambda\left(x_{1}, x_{2}\right) \in K_{1} \times K_{2}\right\} \\
& \leq \sup \left\{\lambda \in \mathbb{R}_{+} \mid \lambda x_{i} \in K_{i}\right\}=j_{K_{i}}\left(x_{i}\right) .
\end{aligned}
$$

to show that the interaction term is $V_{1,2}\left(x_{1}, x_{2}\right)=-\min \left\{h_{i}\left(x_{i}\right) \mid i=1,2\right\}$ and therefore non-zero if $x \neq 0$.

Remark. The symplectic product is also non-trivial for the theory of symplectic capacities in the sense that in general there is no estimate $B(r) \subset D \subset Z(r)$ for $D=$ $K_{1} \times K_{2}$. For all sets $D$ with such an estimate, capacity is calculated trivially from the axioms:

$$
c(B(r))=\pi r^{2} \leq c(D) \leq c(Z(r))=\pi r^{2},
$$

yielding $c(D)=\pi r^{2}$. All examples (ellipsoid, ball and cylinder) whose capacity could be calculated before $[\mathrm{K} 90]$ were of this trivial kind.

\section{References}

[A84] J. P. Aubin, L'analyse nonlinéaire et ses motivations économiques, Masson, Paris, 1984. 
[Cl80] F. H. Clarke, The Erdmann condition and Hamiltonian inclusions in optimal control and the calculus of variations, Canad. J. Math. 32 (1980), 494-509.

[CE80] F. H. Clarke and I. Ekeland, Hamiltonian trajectories with prescribed minimal period, Comm. Pure Appl. Math. 33 (1980), 103-116.

[Cl81] - Periodic solutions to Hamiltonian inclusions, J. Differential Equations 40 (1981), $1-6$.

[CW81] B. C. Croke and A. Weinstein, Closed curves on convex hypersurfaces and period of nonlinear oscillations, Invent. Math. 64 (1981), 199-202.

[C77] J. P. Crouzeix, Contribution à l'étude des fonctions quasiconvexes, Thèse de l'Université Clermont-Ferrand II, 1977.

[C89] —, private communication, 1989.

[E90] I. Ekeland, Convexity methods in Hamiltonian mechanics, Springer, Berlin, 1990.

[EH89] I. Ekeland and H. Hofer, Symplectic topology and Hamiltonian dynamics I, Math. Z. 200 (1989) 355-378; see also C. R. Acad. Sci. Paris Sér. I 307 (1988), 37-40.

[EH90] - - -, Symplectic topology and Hamiltonian dynamics II, Math. Z. 203 (1990), $553-567$.

[El87] Y. Eliashberg, A theorem on the structure of wave fronts and its applications to symplectic topology, Functional. Anal. and Appl. 21 (1987), 227-232.

[G85] M. Gromov, Pseudoholomorphic curves in symplectic manifolds, Invent. Math. 82 (1985), 307-347.

[HZ90] H.W. Hofer and E. Zehnder, A new capacity for symplectic manifolds, in: Analysis et cetera, Academic Press, 1990, 405-429.

[K90] A. F. Künzle, Une capacité symplectique pour les ensembles convexes et quelques applications, Ph.D. thesis, Université Paris IX Dauphine, June 1990.

[K91] - , The least characteristic action as symplectic capacity, preprint, Forschungsinstitut für Mathematik, ETH Zürich, May 1991.

[K94] -, On the minimal number of closed characteristics on hypersurfaces diffeomorphic to a sphere, preprint, Max-Planck-Institut, Bonn, no. 94-4.

[K95] - On the symplectic capacities extending the least characteristic action of convex sets, preprint EPFL, August 1995.

[K95a] - The symplectic product of hypersurfaces, in preparation.

[P99] H. Poincaré, Les méthodes nouvelles de la mécanique céleste, tome III, Paris, 1899.

[R70] R. T. Rockafellar, Convex Analysis, Princeton University Press, Princeton, N.J., 1970 .

[R81] —, The Theory of Subgradients and its Applications to Problems of Optimization. Convex and Nonconvex Functions, Heldermann, Berlin, 1981.

[R89] —, manuscript, Varenna, June 1989.

[Si90] J. C. Sikorav, Systèmes Hamiltoniens et topologie symplectique, Lecture notes, Dipartimento di Matematica dell'Università di Pisa, August 1990.

[V87] C. Viterbo, A proof of Weinstein's conjecture in $\mathbb{R}^{2 n}$, Ann. Inst. H. Poincaré Anal. Non Linéaire 4 (1987), 337-357. 